\title{
Abundances and Energy Spectra of Corotating Interaction Region Heavy Ions
}

\author{
G. M. Mason ${ }^{\mathrm{a}}$, R. A .Leske ${ }^{\mathrm{b}}$, M. I. Desai ${ }^{\mathrm{c}}$, J. R. Dwyer ${ }^{\mathrm{d}}$, J. E. Mazur ${ }^{\mathrm{e}}$, \\ R. A. Mewaldt ${ }^{b}$, R. E. Gold ${ }^{\mathrm{a}}$, and S. M. Krimigis ${ }^{\mathrm{a}}$ \\ ${ }^{a}$ Johns Hopkins Univ. /Applied Physics Lab., Laurel, MD 20723 \\ ${ }^{b}$ California Institute of Technology, Pasadena CA 91125 \\ ${ }^{c}$ Southwest Research Institute, San Antonio TX 78228 \\ ${ }^{d}$ Florida Institute of Technology, Melboume FL 32901 \\ ${ }^{e}$ The Aerospace Corporation, Chantilly VA 20151
}

\begin{abstract}
We have surveyed He-Fe spectra for 41 Corotating Interaction Regions (CIRs) from 1998-2007 observed on ACE. The spectra are similar for all species, and have the form of broken power laws with the spectral break occurring at a few MeV/nucleon. Except for overabundances of $\mathrm{He}$ and $\mathrm{Ne}$, the abundances are close to those of the solar wind. We find the rare isotope ${ }^{3} \mathrm{He}$ is enhanced in $\sim 40 \%$ of the events. In individual CIRs the $\mathrm{Fe} / \mathrm{O}$ ratio correlates strongly with the solar wind $\mathrm{Fe} / \mathrm{O}$ ratio measured 2-4 days prior to the CIR passage. Taken together with previously reported observations of pick-up $\mathrm{He}^{+}$in CIRs, these observations provide evidence that CIRs are accelerated out of a suprathermal ion pool of heated solar wind ions, pick-up ions, and remnant suprathermal ions from impulsive solar energetic particle (SEP) events.
\end{abstract}

Keywords: Interplanetary physics -- Particle acceleration -- Corotating streams -- Energetic particles -- Pickup ions

PACS: $96.50 .-\mathrm{e}, 96.50 . P \mathrm{w}, 96.50 . \mathrm{Qx}, 96.50 . \mathrm{Vg}, 96.50 . \mathrm{Ya}$

\section{INTRODUCTION}

Corotating Interaction Regions (CIRs) are caused by the interaction of slow- and fast-solar wind streams in the inner heliosphere. The stream interaction leads to turbulence and eventually the formation of shocks that propagate away from the stream interface in the forward (outward) and reverse (inward) directions. Figure 1 sketches typical CIR geometry along with the associated plasma and magnetic field signatures (figure from [1]; for recent reviews see [2]).

Energetic ions accelerated in CIRs can give insight into the acceleration and transport processes in these events through the properties of temporal behavior, spectral form, and composition.

\section{OBSERVATIONS}

We have used advanced instruments on the Advanced Composition Explorer spacecraft to carry out a survey of CIRs with precision and sensitivity greatly exceeding previous studies [3-5]. Figure 2 shows a typical event lasting several days

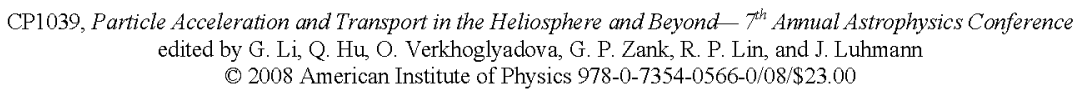




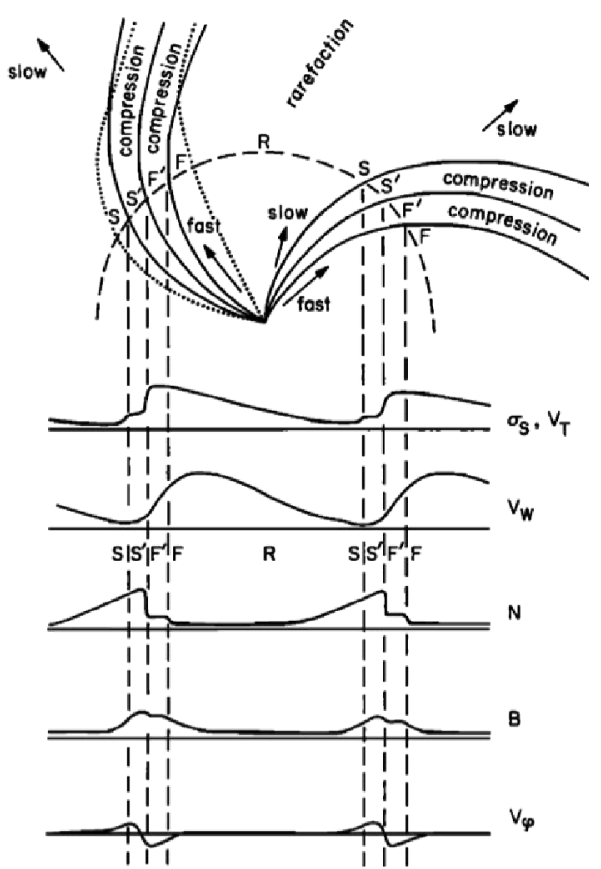

FIGURE 1. Typical geometry of CIRs in the ecliptic plane (top), and (lower traces) solar wind proton thermal speed, radial bulk speed, number density. B is magnetic field intensity; bottom trace is component of solar wind tangential to radial direction (from [1]).

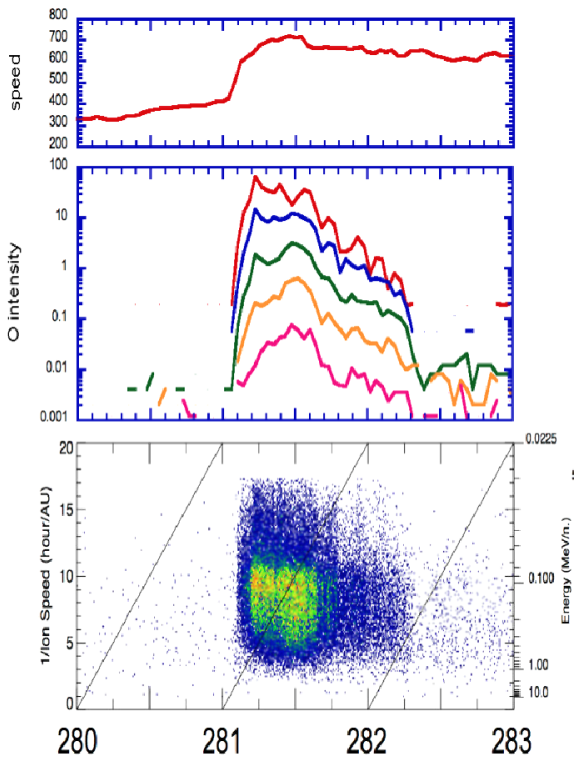

FIGURE 2. Oct. 8, 2005 CIR event observed on ACE; Top: solar wind speed; Middle: $\mathrm{O}$ intensity from 48 to $770 \mathrm{keV} / \mathrm{n}$ with traces separated by a factor of 2 in energy; Bottom: dispersionless ion arrival spectrogram. (Figs. 29 adapted from [7]).

and associated with a solar wind speed increase (top panel [6]), hourly average $O$ intensities, and particle arrival spectrogram showing a dispersionless time history [from 7] . In order to obtain good statistical accuracy for ion measurements, events were selected only if the $230-320 \mathrm{keV} / \mathrm{nuc}$ He intensity exceeded 10 particles/ $\left(\mathrm{s} \mathrm{cm}^{2} \mathrm{sr}\right.$ $\mathrm{MeV} / \mathrm{n}$ ) and lasted at least 24 hours. Plasma and magnetic field signatures were also consulted in this selection, and events were not used if there was possible contamination from solar energetic particle (SEP) events. A total of 41 events were found; we note that many more CIRs occurred during the survey period that did not meet these selection criteria.

Composition and spectral information was obtained by summing over each event; from figure 2 it is clear that the peak intensity period will dominate. The left panel of figure 3 shows mass peaks for the sum of all the events. The major elements are clearly resolved, and the statistical accuracy is excellent. The abundances for each event were obtained by summing over the mass peaks in each case. The right panel of figure 3 shows the He isotopes region. ${ }^{3} \mathrm{He}$ is clearly present in the sum of all the data. Examining histograms such as shown in figure 3 for each individual CIR, we find that 16 of the $41(\sim 40 \%)$ CIRs had finite ${ }^{3} \mathrm{He}$, with enhancements from $\sim 2$ to 6 times the solar wind value. 

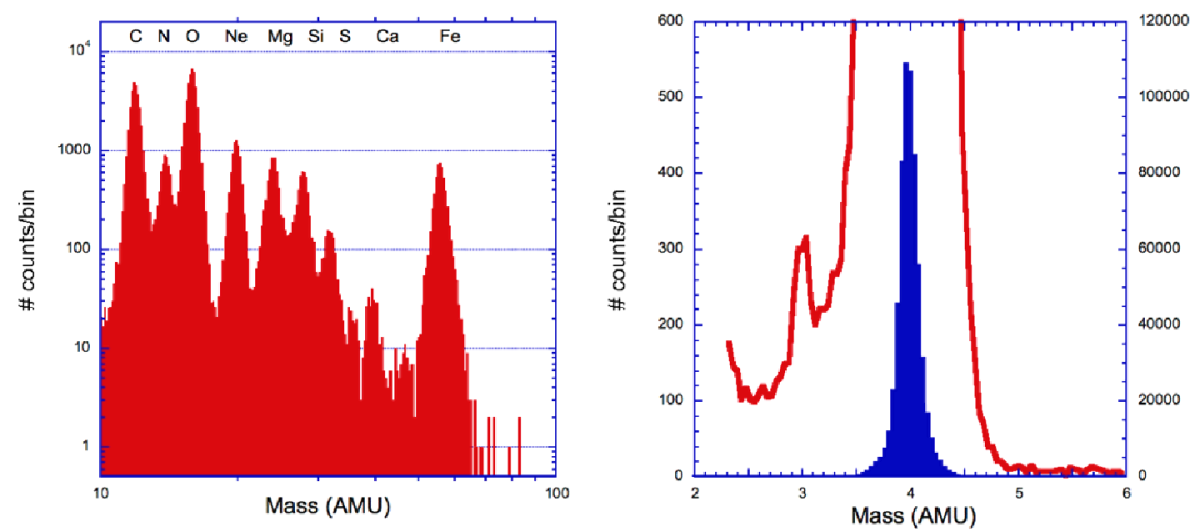

FIGURE 3. Left: mass histograms of $0.32-0.45 \mathrm{MeV} /$ nucleon C-Fe for all events in survey; Right: 0.5-2.0 MeV/n He isotope region showing ${ }^{4} \mathrm{He}$ peak (right scale) and greatly expanded left scale to show ${ }^{3} \mathrm{He}$ peak.

Figure 4(left) shows spectral forms for major elements from the March 22, 2000 CIR, using data from both the ACE ULEIS and SIS instruments. The spectra are well described as broken power laws, with a relatively hard spectrum at low energies which then steepens significantly above a few MeV/nucleon. Note that the high energy portions of the spectra remain power laws up until energies where they intersect the spectra of galactic and anomalous cosmic rays above $30 \mathrm{MeV} /$ nucleon. The event shown in the figure is one of the most intense of the survey; in many cases the spectra were not measured above a few MeV/nucleon due to low intensity.

Figure 4(right) shows ratios of each element with respect to $\mathrm{O}$. In order to separate the data points in the figure, the ratios $\mathrm{He} / \mathrm{O}, \mathrm{Ne} / \mathrm{O}, \mathrm{Mg} / \mathrm{O}, \mathrm{Si} / \mathrm{O}, \mathrm{S} / \mathrm{O}, \mathrm{Ca} / \mathrm{O}$, and $\mathrm{Fe} / \mathrm{O}$ have been multiplied by $10^{-X}$ where $X=2,1,2,3,3,3$, and 5 , respectively. Note that
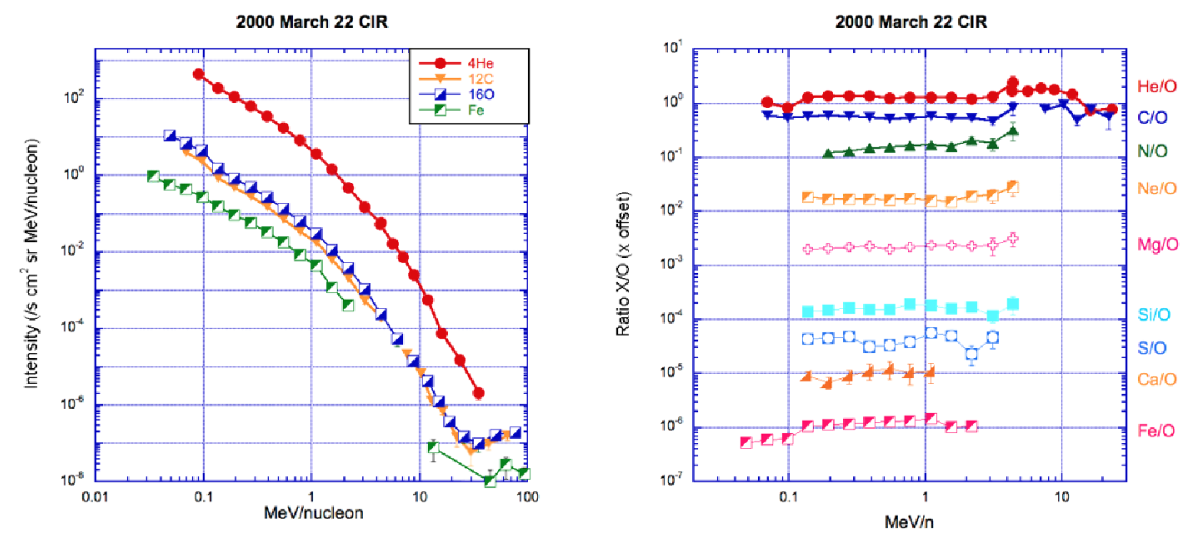

FIGURE 4. Left: spectra for the March 22, 2000 event showing rollovers at a few MeV/nucleon to steeper power laws which continue to $\sim 30 \mathrm{MeV} / \mathrm{nuc}$ where they meet spectra of galactic and anomalous cosmic rays; Right: ratios of elements with respect to $\mathrm{O}$; traces have been offset by factors to avoid crowding (see text). Note ratios are constant to within a factor of $\sim 2$ even though the intensities (left panel) vary over a factor of $\sim 10^{8}$. 

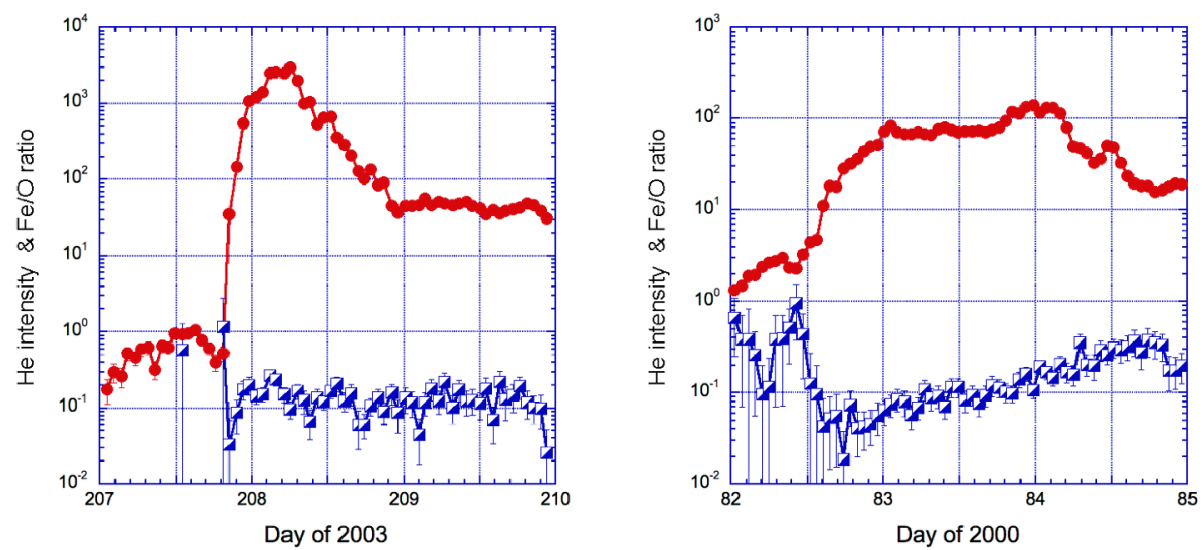

FIGURE 5. Left: hourly average $0.32-0.45 \mathrm{MeV} /$ nuc $\mathrm{He}$ intensity (filled circles) and $\mathrm{Fe} / \mathrm{O}$ ratio (half-filled squares) for the July 26, 2003 event, showing typical behavior where $\mathrm{Fe} / \mathrm{O}$ is constant throughout; Right: March 22, $2000 \mathrm{CIR}$ where the Fe/O showed a highly unusual systematic increase after the event onset. He intensity units are particles $/ \mathrm{s} \mathrm{cm}^{2} \mathrm{sr} \mathrm{MeV} /$ nuc.

the abundance ratios are nearly constant even though the particle intensities vary by a large factor over the energy range shown.

Figure 5 explores the time dependence of abundances within individual CIR events using the $\mathrm{Fe} / \mathrm{O}$ ratio since these two species have significant differences in charge to mass ratio [8] and so could be expected to be sensitive indicators. Figure 5(left) shows typical behavior where the $\mathrm{Fe} / \mathrm{O}$ is essentially constant throughout the event; a counter-example in the right panel was very unusual.

The near constancy of the abundance ratios as a function of energy (Figure 4) and time (Figure 5) contrasts strikingly with the situation in SEP events where spectral forms for different species are often markedly different $[9,10]$ and ratios such as $\mathrm{Fe} / \mathrm{O}$ often show strong, systematic variations [11]. For this reason it is reasonable to expect that the average abundances determined during CIRs are more straightforward to interpret and to compare with other samples of matter. We determined average CIR abundances taking unweighted averages of the ratio of each species to $\mathrm{O}$ for each of the 41 events. Table 1 lists the resulting abundances and compares them with CIR abundances measured at higher energies and with and fast- and slow-solar wind.

Figure 6 plots the CIR average abundances along with average abundances from fast-and slow-solar wind streams. Both He and Ne are overabundant in the CIRs as been seen in previous studies [e.g., 12]. For the rest of the elements the overall best agreement is with the fast solar wind.

Although the mean CIR average abundances shown in Table 1 have dispersions of order 5-10\%, individual CIRs differ much more than this as shown in Figure 7, which is a plot of element ratios in each CIR relative to O, plotted vs. the $\mathrm{Fe} / \mathrm{C}$ ratio for the same CIR. Thus, each of the 41 events has a single $\mathrm{Fe} / \mathrm{C}$ value with its respective $\mathrm{He} / \mathrm{O}, \mathrm{N} / \mathrm{O}$, etc. plotted. It can be seen that the $\mathrm{Fe} / \mathrm{C}$ ratio varies by almost an order of magnitude amongst the 41 events, although if the two lowest Fe/C cases are removed, the remaining events show a variation of a factor of $\sim 5$. It is also clear that the abundance variations are correlated in that events with high $\mathrm{Fe} / \mathrm{C}$ also tend to show high $\mathrm{Si} / \mathrm{O}$, etc. $\mathrm{He} / \mathrm{O}$ and $\mathrm{Ne} / \mathrm{O}$ are slightly anticorrelated with $\mathrm{Fe} / \mathrm{C}$. 
TABLE 1. Mean Heavy Ion Abundances for CIRs and Solar Wind

\begin{tabular}{|c|c|c|c|c|}
\hline Element & $\begin{array}{l}\text { CIRs }^{\mathrm{a}} \quad(0.385 \\
\mathrm{MeV} \text { nucleon }\end{array}$ & $\begin{array}{c}\operatorname{CIRs}^{\mathrm{b}}(5-12 \\
\mathrm{MeV} \text { nucleoon } \\
-1\end{array}$ & $\begin{array}{l}\text { Slow SW }{ }^{c}(\sim 1 \\
\left.\text { keV nucleon }{ }^{-1}\right)\end{array}$ & $\begin{array}{l}\text { Fast SW }{ }^{\mathrm{c}}(\sim 2 \\
\mathrm{keV} \text { nucleon } \\
-1)\end{array}$ \\
\hline${ }^{4} \mathrm{He} \ldots \ldots$. & $273 \pm 72$ & $159 \pm 10$ & $95.9 \pm 28.8$ & $72.7 \pm 21.8$ \\
\hline $\mathrm{C} \ldots \ldots \ldots$ & $0.760 \pm 0.023$ & $0.890 \pm 0.036$ & $0.670 \pm 0.067$ & $0.710 \pm 0.080$ \\
\hline $\mathrm{N} \ldots \ldots \ldots$ & $0.143 \pm 0.005$ & $0.140 \pm 0.014$ & $0.069 \pm 0.021$ & $0.143 \pm 0.016$ \\
\hline $\mathrm{O} \ldots \ldots \ldots$ & $1.000 \pm 0.020$ & $1.000 \pm 0.037$ & $\equiv 1$ & $\equiv 1$ \\
\hline $\mathrm{Ne} \ldots . .$. & $0.206 \pm 0.009$ & $0.170 \pm 0.016$ & $0.091 \pm 0.027$ & $0.071 \pm 0.010$ \\
\hline $\mathrm{Mg} . . . .$. & $0.148 \pm 0.006$ & $0.140 \pm 0.014$ & $0.147 \pm 0.030$ & $0.108 \pm 0.022$ \\
\hline Si........ & $0.095 \pm 0.005$ & $0.100 \pm 0.012$ & $0.167 \pm 0.034$ & $0.088 \pm 0.014$ \\
\hline $\mathrm{S} \ldots \ldots \ldots$ & $0.028 \pm 0.002$ & $0.050 \pm 0.008$ & $0.049 \pm 0.010$ & $0.035 \pm 0.004$ \\
\hline $\mathrm{Ca} \ldots \ldots$ & $0.007 \pm 0.001$ & & $0.017 \pm 0.003$ & $0.007 \pm 0.003$ \\
\hline Fe........ & $0.088 \pm 0.007$ & $0.097 \pm 0.011$ & $0.120 \pm 0.024$ & $0.067 \pm 0.007$ \\
\hline
\end{tabular}

Table notes: a: [7]; b: [13]; c: $[14,15]$

These basic trends are similar to systematic variations seen in SEPs $[16,17]$, but are much smaller.

The factor of $\sim 5$ range of $\mathrm{Fe} / \mathrm{O}$ variations shown in Figure 7 is similar to shorterterm variations in the $\mathrm{Fe} / \mathrm{O}$ ratio in the solar wind. To explore whether $\mathrm{Fe} / \mathrm{O}$ in individual CIRs was related to the solar wind $\mathrm{Fe} / \mathrm{O}$ ratio measured near the time of the CIR, we calculated the correlation coefficient between the $41 \mathrm{CIR}$ values and the solar wind Fe/O ratio measured by the ACE/SWICS instrument [18]. The solar wind Fe/O was measured over a time period identical in length to the time interval used for the energetic particles, but shifted prior to the energetic particle observations by 0 to 10

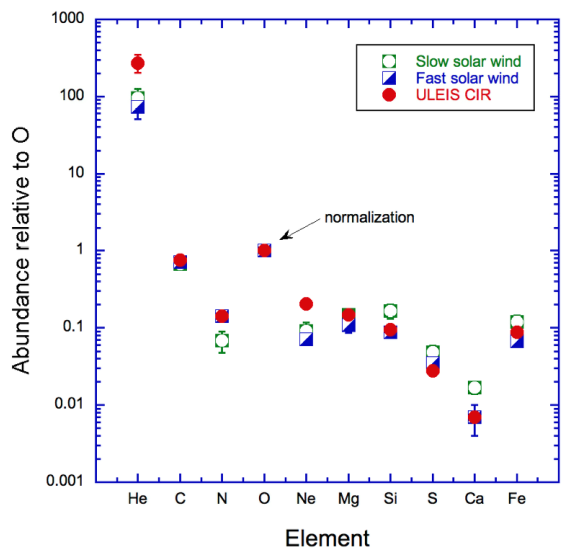

FIGURE 6. $0.32-0.45 \mathrm{MeV} / \mathrm{nuc}$ CIR abundances compared to solar wind from slow- and fast solar wind streams.

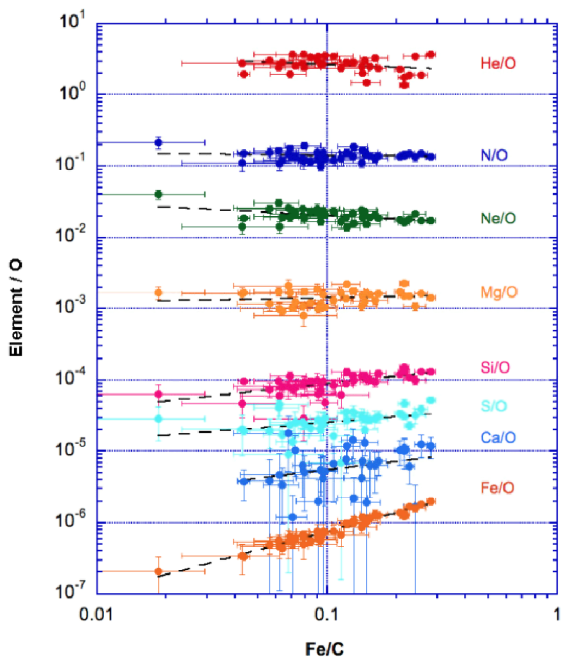

FIGURE 7. Average abundances in each of the 41 CIRs normalized to $\mathrm{O}$ plotted vs $\mathrm{Fe} / \mathrm{C}$. Offset factors for each element are same as in Figure 4 (right panel). 

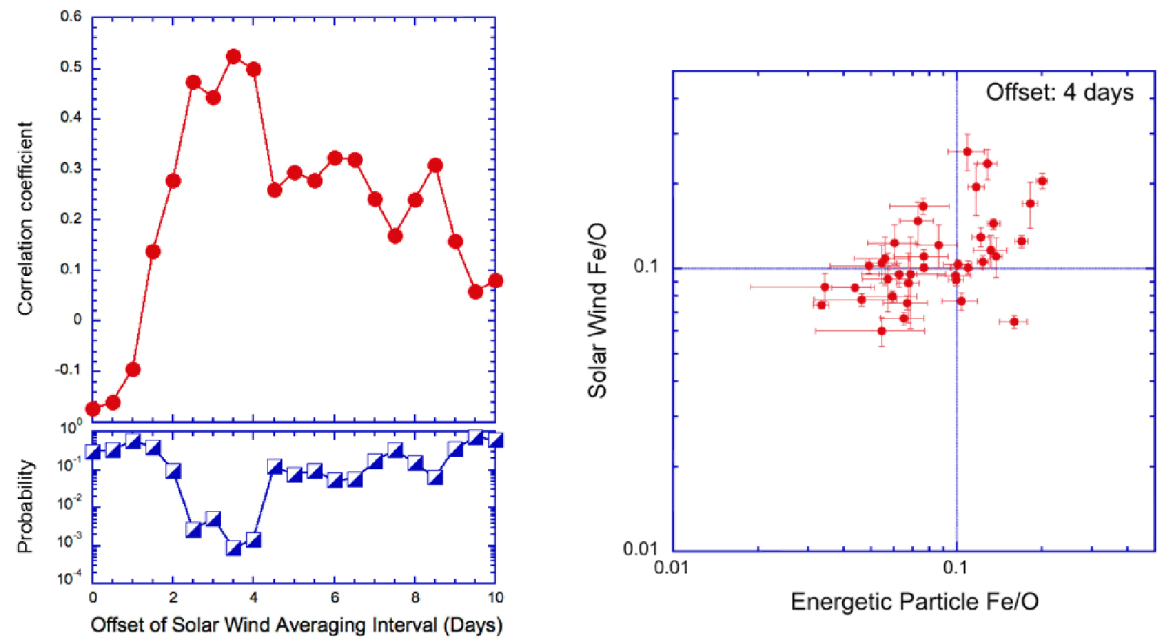

FIGURE 8. Left, upper panel: correlation coefficient between $\mathrm{Fe} / \mathrm{O}$ in the $41 \mathrm{CIRs}$ and $\mathrm{Fe} / \mathrm{O}$ in solar wind measured 0-10 days prior to the CIR passage; Left, lower panel: probability that the correlation coefficient could arise from random data; Right: individual points for solar wind vs. energetic particle $\mathrm{Fe} / \mathrm{O}$ for the case of 4.0 day offset.

days. Figure 8(left) shows the result, where the correlation coefficient is low for 0 offset, but grows to a significant value for offsets of 2-4 days, and then declines again. The lower left panel shows the probability that the correlation could arise from random data, and for the 2-4 day offsets, the probability is very small. Figure $8($ right $)$ shows the set of 41 points for the case of a 4 day offset, where it can be seen that the correlation is based on many of the points and not a couple of outliers.

Figure 9 shows properties of the CIR spectra, where we fitted power laws in kinetic energy over limited energy ranges. In the lowest energy range (0.16-0.91 MeV/nuc, red filled circles) the average slope is $2.51 \pm 0.10$, but the range of values is $\sim 1.3$ to 4 . The $\mathrm{He}$ and $\mathrm{O}$ slopes are nearly identical. In the 0.91-3.6 MeV/nuc range, the slopes steepen by roughly 2 units with an average value of $4.47 \pm 0.17$. For 3.4-9.7 $\mathrm{MeV} / \mathrm{nuc}$ the slopes steepen by another $\sim 2$ units, and are only determined in events with harder spectra at low energies.

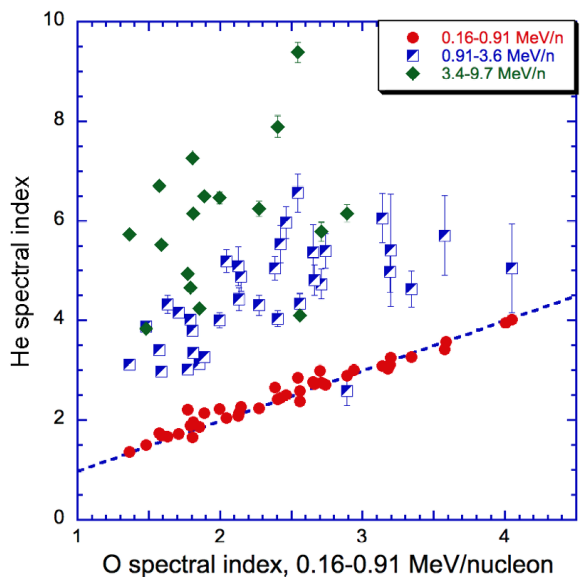

FIGURE 9. He spectral index $\gamma$, for kinetic energy power law spectral shape over three energy ranges vs. O spectral index in range 0.16$0.91 \mathrm{MeV} / \mathrm{nuc}$. Dashed line is for equal He and O spectral slopes at low energy. 


\section{DISCUSSION}

\section{Acceleration Mechanisms}

The features of CIRs measured in this survey fit well into the prior general models for these events such as the Fisk and Lee model [19], but provide new constraints on the acceleration mechanism and properties of the seed population. The broad energy range and high statistical precision of the ACE measurements allow much more stringent tests of spectral forms than previously possible. Figure 10 compares the $\mathrm{He}$ spectrum from the March 22, 2000 CIR (see Fig. 4) with differential kinetic energy spectral forms from three models. Fig. 10(a) shows the fit to the Fisk and Lee [19] form $d J / d E=J_{0} v^{-n} \exp \left(v / v_{0}\right)$, where $d J / d E$ is the differential intensity, $v$ is the particle speed, and $J_{0}, n$ and $v_{0}$ are adjusted in the fit. Fig. 10(b) shows the fit to the Jones and Ellison [20] form $d J / d E=N_{0} E^{-\gamma} \exp \left(-E / E_{0}\right)$, where $E$ is kinetic energy per nucleon, and $N_{0}, \gamma$ and $E_{0}$ are adjusted. Figure 10(c) shows a comparison with the spectral form suggested by Fisk and Gloeckler [21] for a mechanism that did not specifically address CIRs but which might be suitable due to their nearly steady-state. For Fig. 10(c) the spectral form is $d J / d E=N_{0} E^{-1.5} \exp \left(-E / E_{0}\right)$, and we note that in this case there is one less adjustable parameter than in the other cases.

At low energies each of the spectral forms gives a reasonably good fit to the data, but the model spectra used were those at the CIR and do not include the results of propagation in to $1 \mathrm{AU}$ which would strongly affect the data below $\sim 100 \mathrm{keV} / \mathrm{nuc}$ [22]. The presence of CIR spectral power laws down to solar wind energies [23] suggests that the low energy particles are energized closer to $1 \mathrm{AU}$ than the $\sim 3-4 \mathrm{AU}$ distances [24] where the CIR intensities peak. This is also suggested by the observed 2-4 day peak correlation between the Fe/O in the solar wind the CIR, which for slow solar wind implies an origin at $r \sim 1.3-2 \mathrm{AU}$ for these particles.

Above $\sim 10 \mathrm{MeV} / \mathrm{nuc}$ the CIR spectrum is harder than the fits. The presence of power laws in the data out to high energies was typical in those events intense enough to allow high energy measurements. In the case of the CIR models used here [19, 20] this problem might be due to the use of a seed population which was a delta-function in energy. If a more realistic power law seed population were used [25], this might
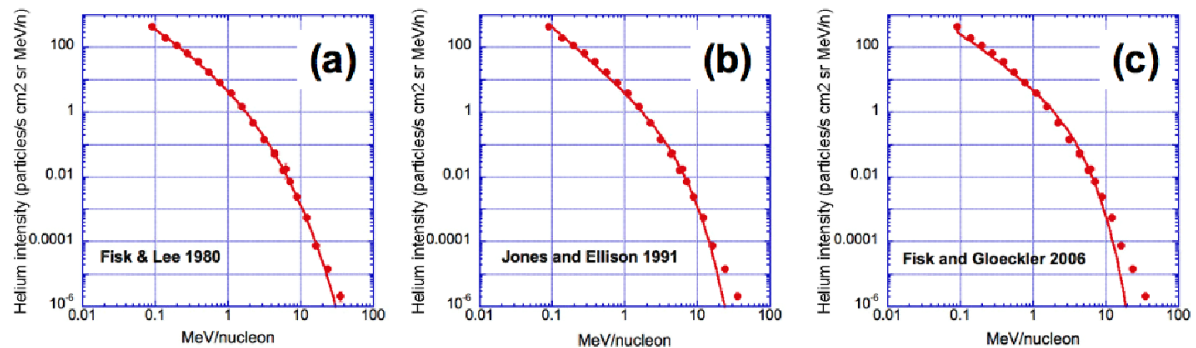

FIGURE 10. Fits to the He spectrum from the March 22, 2000 CIR using the functional forms of models from $(a)$ Fisk and Lee (1980), (b) Jones and Ellison (1991), and (c) Fisk and Gloeckler (2006). Note that above $10 \mathrm{MeV} / \mathrm{nuc}$ the CIR spectrum is harder than the exponential fall off in each of the models. Of the three model spectral forms, the one from Fisk and Lee gives the best fit. 
produce a harder spectral form at high energies. We note that the deduced source of low energy CIR ions close to $1 \mathrm{AU}$ implies that the particles may be energized in regions before the shocks have formed. Giacalone and Jokipii [26] have shown that in fact shocks are not required for particle energization, but rather that the compression associated with the CIR is sufficient. Spectral forms from their model are also consistent with the observations presented here [7].

\section{Abundances and Seed Population}

Figure 6 and Table 1 above showed that the major elemental abundances in the CIRs are close to solar wind values with the exception of $\mathrm{He}$ and $\mathrm{Ne}$, which are overabundant in CIRs as noted in earlier work. The correlation between individual $\mathrm{CIR} \mathrm{Fe} / \mathrm{O}$ and solar wind $\mathrm{Fe} / \mathrm{O}$ variations shows a close relation between the $\mathrm{CIR}$ particles and the solar wind. However, the presence of enhanced ${ }^{3} \mathrm{He}$ values reported here, along with the routine detection of $\mathrm{He}^{+}$at $10-20 \%$ of $\mathrm{He}^{++}$in CIRs $[8,23,27]$ shows that the bulk solar wind cannot be the only source since ${ }^{3} \mathrm{He}$ and $\mathrm{He}^{+}$are extremely rare in the solar wind. The best overall explanation for the observed elemental, isotopic, and charge state abundances is that the CIRs energize particles from the suprathermal tail, and that this seed particle source consists of heated solar wind, pick-up ions, and remnant ${ }^{3}$ He-rich SEP material. Such a source would be expected to produce CIRs with higher $\mathrm{He}^{+}$abundance beyond 1 AU since the pick-up ion source is relatively more important there, and indeed this is observed [28].

The overabundance of He and Ne in CIRs could suggest a pick-up ion source, but since these elements are highly stripped in CIRs [8, 29] a simple pick-up ion explanation is ruled out. For additional comments on the He abundance see [30].

\section{C/O in CIRs, SEPs, and IP shocks}

The CIR C/O ratio has long been considered anomalous since it is clearly higher than $\mathrm{C} / \mathrm{O}$ in SEPs and in solar material $[31,32]$. Before comparing the CIR C/O ratio with other matter samples, it is useful to compare the current survey with other results. Figure 11 shows CIR C/O as a function of the energy (left) and year of observation (right) from the current and prior surveys [7, 31-38]. The different surveys show a large spread, but there is no evident trend with energy. Plotting the data vs. year of observation (Fig. 11 right) reveals that the earlier measurements tended to show higher $\mathrm{C} / \mathrm{O}$, and were mostly taken during periods of low sunspot number. The correlation coefficient between the $\mathrm{C} / \mathrm{O}$ ratio and year of observation is 0.28 , which for 13 observations has a $35 \%$ chance of being random. To examine this further, the figure also shows the individual $\mathrm{C} / \mathrm{O}$ ratios from the 41 events in the current survey (open circles) which show a broad spread but no clear trend with sunspot number. Looking at the spread of $\mathrm{C} / \mathrm{O}$ values in the current survey, and considering the small number of events or limited statistical accuracy in the prior surveys, we suggest that the differences in $\mathrm{C} / \mathrm{O}$ amongst the surveys is mostly a sampling effect. Taking together that charge resolution of the ACE instruments is as good or better than the earlier instruments, the statistical precision is much higher, and 

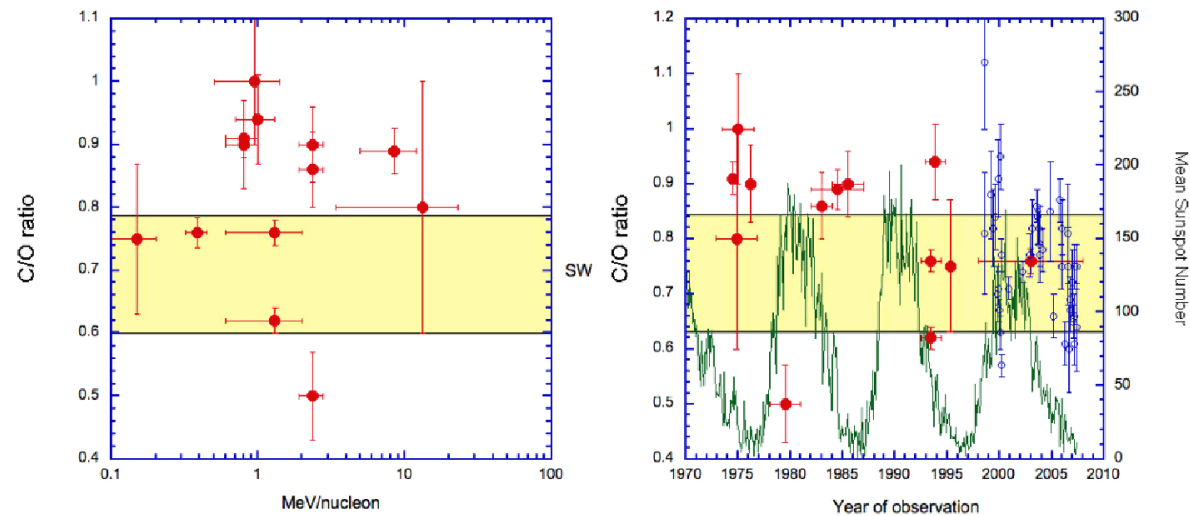

FIGURE 11. Left: $\mathrm{C} / \mathrm{O}$ ratio from current and previous surveys vs. energy/nucleon; Right: $\mathrm{C} / \mathrm{O}$ from current and previous surveys (filled circles) vs. year of observation. Green line shows NOAA sunspot numbers. Open circles: $\mathrm{C} / \mathrm{O}$ from each of the $41 \mathrm{CIRs}$ in the current survey. Yellow shaded area: range of fast- and slow-solar wind $\mathrm{C} / \mathrm{O}$ from Table 1.

the number of CIRs with abundance determinations is larger than prior surveys, we believe that the current survey represents the most reliable determination of $\mathrm{C} / \mathrm{O}$ in CIRs.

Figure 12 compares CIR C/O with fast- and slow- solar wind, IP shocks [39], large SEP events [17], impulsive SEP events [40], the solar photosphere [41], and the solar corona [42]. The CIR C/O is consistent with the solar wind value which in turn is somewhat higher than the photosphere and coronal values. However, the $\mathrm{C} / \mathrm{O}$ which stands out as puzzling are the solar particle event and IP shock values, which are notably lower. It is difficult to imagine that this difference between CIR and SEP C/O is due to the acceleration mechanism since the $\mathrm{C} / \mathrm{O}$ ratios differ by a factor of $\sim 2$ while the charge to mass ratios of the species are quite similar. This points to the seed population as the cause of the difference, implying that the acceleration sites of SEPs have systematically lower $\mathrm{C} / \mathrm{O}$ than the solar wind [17]. This implies that traveling IP shocks must also energize particles quite close to the Sun where $\mathrm{C} / \mathrm{O}$ is low (i.e. not the solar wind value). Thus, differences between CIR and SEP abundances are due to the seed population, and IP shocks appear to re-

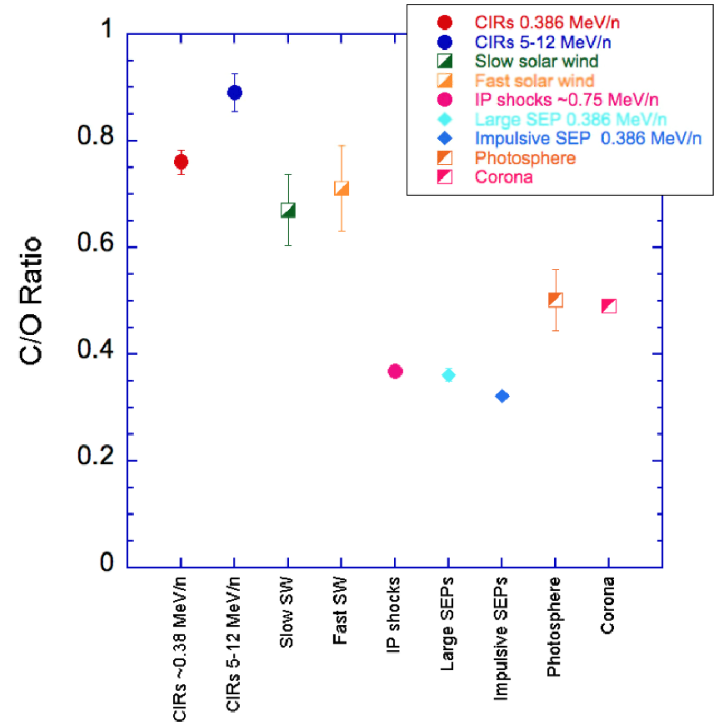

FIGURE 12. Comparison of CIR abundances at low and high energies with other samples of matter. 
accelerate previously energized SEP particles $[43,44]$ close to the Sun.

\section{ACKNOWLEDGMENTS}

This work was supported in part by NASA under grant NNG04GJ51G at the Johns Hopkins University/Applied Physics Laboratory. We thank the following organizations for permission to publish copyrighted material: American Geophysical Union (Fig. 1, from [1]); and American Astronomical Society (Figs. 2-9, from [7]).

\section{REFERENCES}

1. Belcher, J.W. and L. Davis, Jr., J. Geophys. Res., 76: 3534 (1971).

2. Balogh, A., et al., eds. Space Science Series of ISSI. Kluwer Academic Publishers: Dordrecht, (1999).

3. Mason, G.M., et al., Space Sci. Rev., 86: 409-448 (1998).

4. Stone, E.C., et al., Space Sci. Rev., 86: 357-408 (1998).

5. Stone, E.C., et al., Space Sci. Rev., 86: 1-22 (1998).

6. McComas, D.J., et al., Space Sci. Rev., 86: 563-612 (1998).

7. Mason, G.M., et al., Astrophys J, 678: 1458-1470 (2008).

8. Möbius, E., et al., Geophys. Res. Lett., 29: 2001GL013410 (2002).

9. Cohen, C.M.S., et al., J. Geophys. Res., 110: A09S16 doi: 10.1029/2005JA011004 (2005).

10. Mewaldt, R.A., et al., AIP CP781: 227-232 (2005).

11. Mason, G.M., et al., Astrophys. J. Lett., 647: L65-L68 (2006).

12. Gloeckler, G., Rev. Geophys. Space Phys., 17: 569-586 (1979).

13. Reames, D.V., Adv. Space Res., 15: 41 (1995).

14. von Steiger, R., et al., J. Geophys. Res., 105: $27217-27238$ (2000).

15. Gloeckler, G. and J. Geiss, Space Sci. Rev, 130: 139-152 (2007).

16. Breneman, H.H. and E.C. Stone, Astrophys. J. (Letters), 299: L57-L61 (1985).

17. Desai, M.I., et al., Space Sci. Rev, 124: 261-285 (2006).

18. Gloeckler, G., et al., Space Sci. Rev., 86: 497-539 (1998).

19. Fisk, L.A. and M.A. Lee, Astrophys. J., 237: 620-626 (1980).

20. Jones, F.C. and D.C. Ellison, Space Sci. Rev., 58: 259 (1991).

21. Fisk, L.A. and G. Gloeckler, Astrophys J Letters, 640: L79-L82 (2006).

22. Mason, G.M., et al., Space Sci. Rev., 89: 327-367 (1999).

23. Chotoo, K., et al., J. Geophys. Res., 105: 23107-23122 (2000).

24. Van Hollebeke, M.A.I., et al., J. Geophys. Res., 83: 4723 (1978).

25. Gloeckler, G., in A.I.P. Conf. Proc. \#679, M. Velli, et al., Editors. A.I.P.: New York. p. 583-588, (2003).

26. Giacalone, J., et al., Astrophys. J., 573: 845-850 (2002).

27. Hilchenbach, M., et al., in Solar Wind Nine, S.R. Habbal, et al., Editors. A.I.P.: NY. p. 605-608, (1999).

28. Gloeckler, G., et al., J. Geophys. Res., 99: 17637-17643 (1994).

29. Mazur, J.E., et al., Astrophys. J., 566: 555-561 (2002).

30. Gloeckler, G., et al., AIP CP - this volume, (2008).

31. Scholer, M., et al., Astrophys. J., 227: 323-328 (1979).

32. Reames, D.V., et al., Astrophys. J. (Letters), 382: L43-L46 (1991).

33. Richardson, I.G., et al., J. Geophys. Res., 98: 13-32 (1993).

34. Gloeckler, G., et al., Astrophys. J. (Letters), 230: L191 (1979).

35. McGuire, R.E., et al., Astrophys. J., 224: L87-L91 (1978).

36. Mason, G.M., et al., Astrophys. J. (Letters), 486: L149-L152 (1997).

37. Fränz, M., et al., Geophys. Res. Letters, 26: 17-20 (1999).

38. Reames, D.V., Space Sci. Rev., 90: 413-491 (1999).

39. Desai, M.I., et al., Astrophys. J., 588: 1149-1162 (2003).

40. Mason, G.M., et al., Astrophys. J., 606: 555-564 (2004).

41. Lodders, K., Astrophys J, 591: 1220-1247 (2003).

42. Feldman, U. and K.G. Widing, Space Sci. Rev, 107: 665-720 (2003).

43. Desai, M.I., et al., Ap.J., 611: 1156-1174 (2004).

44. Mewaldt, R.A., et al., in Geophys. Monograph \#165, N. Gopalswamy, et al., Editors. AGU: Washington. p. 115-126, (2006). 